LAWRENCE LIVERMORE NATIONAL LABORATORY

\title{
LASER PERFORMANCE OPERATIONS MODEL (LPOM)
}

M. J. Shaw, W. H. Williams, R. K. House, C. A. Haynam

January 7, 2004

Photonics West - Lasers and Applications in Science and Technology (LASE) 2004

San Jose, California, January 24-29, 2004 
This document was prepared as an account of work sponsored by an agency of the United States Government. Neither the United States Government nor the University of California nor any of their employees, makes any warranty, express or implied, or assumes any legal liability or responsibility for the accuracy, completeness, or usefulness of any information, apparatus, product, or process disclosed, or represents that its use would not infringe privately owned rights. Reference herein to any specific commercial product, process, or service by trade name, trademark, manufacturer, or otherwise, does not necessarily constitute or imply its endorsement, recommendation, or favoring by the United States Government or the University of California. The views and opinions of authors expressed herein do not necessarily state or reflect those of the United States Government or the University of California, and shall not be used for advertising or product endorsement purposes. 


\title{
Laser Performance Operations Model (LPOM)
}

\author{
Michael Shaw*, Wade Williams, Ronald House, Chris Haynam \\ Lawrence Livermore National Laboratory, Livermore, CA 94550
}

This work was performed under the auspices of the U.S. Department of Energy by University of California, Lawrence Livermore National Laboratory under Contract W-7405-Eng-48.

\begin{abstract}
The Laser Performance Operations Model (LPOM) has been developed to provide real time predictive capabilities for the National Ignition Facility (NIF) at Lawrence Livermore National Laboratory. LPOM uses diagnostic feedback from previous NIF shots to maintain accurate energetics models for each of the 192 NIF beamlines (utilizing one CPU per laser beamline). This model is used to determine the system setpoints (initial power, waveplate attenuations, laser diagnostic settings) required for all requested NIF shots. In addition, LPOM employs optical damage models to minimize the probability that a proposed shot may damage the system. LPOM provides post-shot diagnostic reporting to support the NIF community. LPOM was deployed prior to the first main laser shots in NIF, and has since been used to set up every shot in NIF"'s first quad (four beamlines). Real-time adjustments of the codes energetics parameters allows the LPOM to predict total energies within 5\%, and provide energy balance within the four beamlines to within $2 \%$ for shots varying from 0.5 to $26 \mathrm{~kJ}(1.053 \mu \mathrm{m})$ per beamline. The LPOM has been a crucial tool in the commissioning of the first quad of NIF.
\end{abstract}

Keywords: Solid-state lasers, fusion, controls, feedback, modeling

\section{INTRODUCTION}

The NIF ${ }^{1}$ currently under construction at LLNL will be a U.S. Department of Energy and NNSA national center to study inertial confinement fusion and the physics of extreme energy densities and pressures. It will be a vital element of the NNSA Stockpile Stewardship program (SSP), which ensures the reliability and safety of U.S. nuclear weapons. The SSP will achieve this through a combination of above-ground test facilities and power computer simulations using the NNSA's Accelerated Scientific Computing Initiative (ASCI). In NIF, up to 192 extremely powerful laser beams will compress small fusion targets to conditions in which they will ignite and burn, liberating more energy than is required to initiate the fusion reactions. NIF experiments will allow the study of physical processes at temperatures approaching 100 million $\mathrm{K}$ and 100 billion times atmospheric pressure. These conditions exist naturally only in the interior of stars and in nuclear weapons explosions. The first four of NIF's laser beams (one quad) became operational in late 2002, and have been undergoing commissioning tests for the past year.

Success on many of the NIF laser's missions depends on obtaining precisely specified energy waveforms from each of the 192 beams over a wide variety of pulse lengths and temporal shapes, and producing energy balance among the beams to a very high precision. Since the energetic performance of each of the beams will be different, due to slight differences in amplifier gains and optical transmission losses in the constructed beamline, a computational model of the facility is required to accurately determine the input conditions required to generate the requested output. The model must be part of a computational system connected to the NIF controls system, in order to provide the setup information in a time frame consistent with NIF's shot cycle time. The Laser Performance Operations Model (LPOM) ${ }^{2,3}$ has been developed to provide this function for NIF. The LPOM will be one of the NIF Integrated Computer Control System (ICCS) high-level software supervisors. The primary role of the LPOM is to automate the setup of the 192 individual NIF laser beams. To achieve this, LPOM maintains a current description of the system that includes the optical paths, optical losses, amplifier configurations, and frequency conversion configurations for each beam, as well as a database of diagnostic measurements, laser energy, and power at various locations along the beamline. LPOM uses a detailed energy extraction and propagation code called $\mathrm{PROP}^{4}$, the mainstay of design, verification, and component selection for the NIF laser system, to calculate the energetics throughout the beamlines, based on the current optical properties of each of the 'as-built' NIF beamlines. PROP is a 4-dimensional (3 spatial and 1 temporal) code that models energy extraction from amplifiers by solving the Frantz-Nodvik equation ${ }^{5}$. Propagation of the laser pulse is modeled using a Fast-Fourier Transform method. The optical imperfections in the many components comprising each NIF beamline that significantly 
influence beam propagation. PROP includes these effects either as measured metrology data or as power spectral density-based simulated phase screens. With careful inclusion of this information, a quantitative prediction of the nearfield beam modulation and far-field spot size can be made by LPOM. By maintaining an accurate description of the optical system, and by using a detailed physics model at its core, LPOM can accurately calculate the required settings for the Injection Laser System (ILS) that will produce the requested output energies and powers. In addition to the ILS settings, LPOM also predicts the energies and powers at each of the laser diagnostic locations in NIF, thereby assuring that the each diagnostic is set up to accurately measure the results of each shot.

A second function of the LPOM is to provide online equipment protection of the NIF optical system. After calculating the setpoints for a proposed shot, the LPOM provides an assessment of the shot to the NIF shot director prior to the initiation of the shot. This assessment includes a report on the probability of a shot causing optical damage, as well as a report on the feasibility of achieving the proposed shot goals. The LPOM's shot assessment is part of NIF's "defense-in-depth" approach to equipment protection, playing an administration role in preventing conditions that could lead to significant optical damage. In addition to shot assessment, LPOM provides an additional equipment protection feature after a shot, by verifying that the measured energies and powers match those predicted by the model. The final function of LPOM is to provide post-shot data analysis and reporting to the NIF user community.

In this paper, we begin with a general description of the NIF laser system. We then describe the LPOM, and its role in NIF shot activities. We conclude with a series of comparisons of measured and predicted data from the first six months of NIF commissioning activities.

\section{DESCRIPTION OF NIF}

NIF is shown schematically in Figure 1. NIF consists of four main elements: a laser system and optical components; the target chamber and its experimental systems; an environmentally controlled building housing the laser system and target area; and an integrated computer control system. NIF's laser system features 192 high-power laser beams. A NIF laser beam begins with a nanojoule energy pulse from the master oscillator (MOR), a diode-pumped fiber laser system that can provide a variety of pulse shapes for target experiments. The master oscillator pulse is shaped in time and smoothed in intensity and then transported to the preamplifier modules (PAM) for amplification and spatial beam shaping. Each PAM first amplifies the pulse by a factor of 1 million (to the millijoule level) in a regenerative amplifier. The output pulse from the regenerative amplifier is propagated through a beam shaping module that modifies the output Gaussian spatial shape to carefully shaped pulse that will compensate for non-uniformities in the main laser amplifier gain profiles. The shaped pulse is then amplified once again up to the energies of several Joules by passing the beam four times through a flashlamp-pumped rod amplifier. The beam out of the PAM then passes through the Preamplifier Beam Transport System (PABTS) where a set of waveplates and polarizers splits the energy for injection into four separate main laser beamlines. There are a total of 48 PAMs on NIF, each feeding a 'quad' of four laser beams.

From the PABTS the laser beam next enters the main laser system, which consists of two large amplifier units - the power amplifier and the main amplifier. These amplifier systems are designed to efficiently amplify the nominal 1-J input pulse from the PAM/PABTS to the required power and energy maintaining the input beam's spatial and spectral characteristics. Since the temporal shape of the input beam is modified significantly as it passes through the main laser due to gain saturation, the initial MOR pulse shape has to be designed to compensate for all temporal distortion accrued in the preamplifier and main amplifier sections. The NIF main amplifiers are designed to contain 18 glass slabs per beam line, with 11 slabs in the main amplifier section and 7 slabs in the power amplifier section. The amplifiers use 42-kg slabs, $46 \mathrm{~cm}$ x $81 \mathrm{~cm}$ x $3.4 \mathrm{~cm}$ of neodymium-doped phosphate glass set vertically at Brewster's angle to minimize reflective losses in the laser beam. The slabs are stacked four high and two wide to accommodate a 'bundle' of eight laser beams.

The nominal operating configuration of NIF has a beam pass 4 times through the main amplifier and twice through the power amplifier. Multipassing through the main laser amplifiers is achieved using a kind of optical switch called a plasma electrode Pockels cell (PEPC) ${ }^{6}$. This device uses electrically induced changes in the refractive index of an electrooptic crystal (made of KDP). When combined with a polarizer, the PEPC allows light to pass through or reflect off a polarizer. The PEPC will essentially trap the laser light between two mirrors as it makes two round trips through 
the main amplifier system before being switched out to continue its way back through the power amplifier on its way to the target chamber.

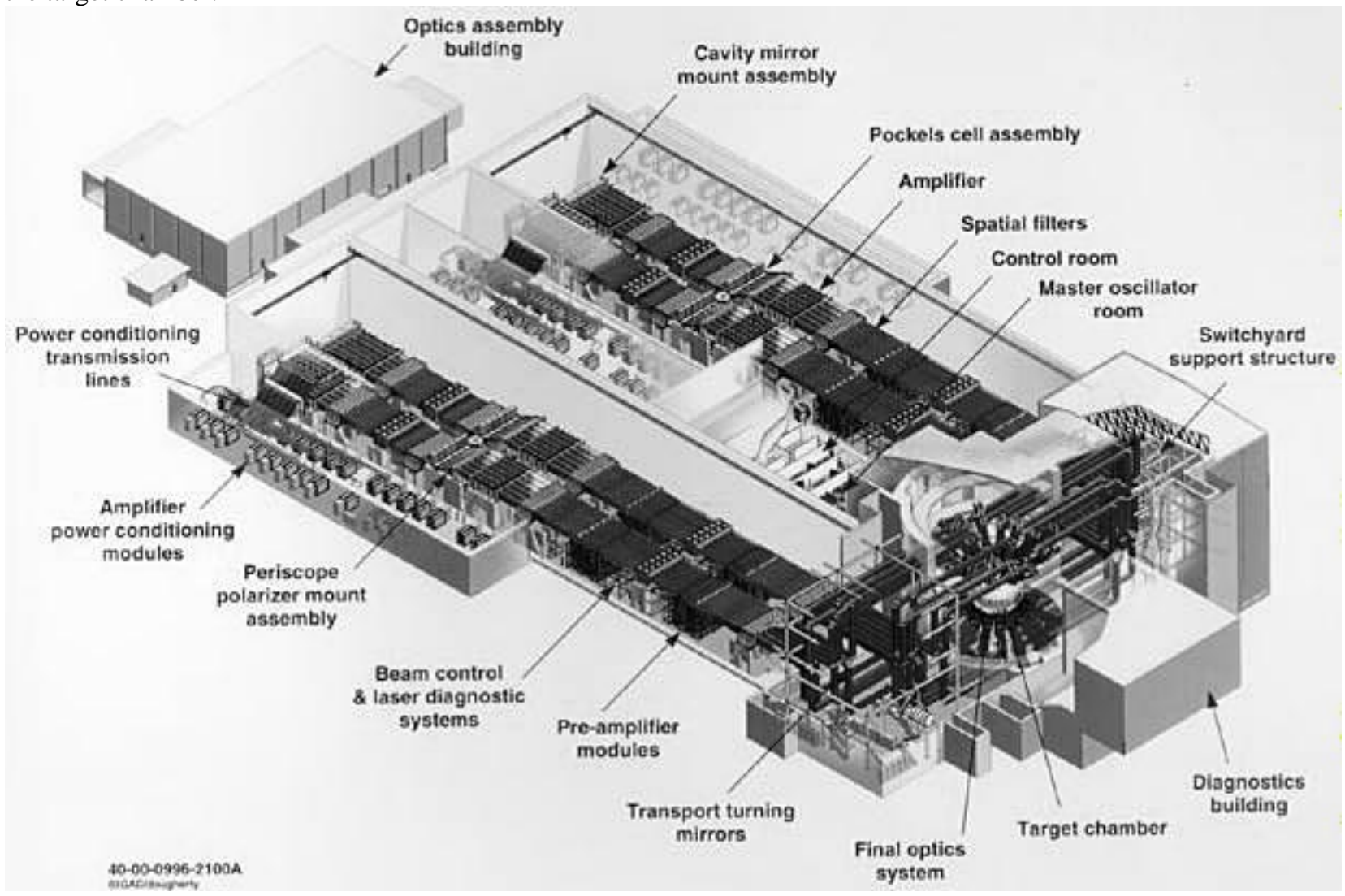

Figure 1: Schematic view of the National Ignition Facility showing the main elements of the laser system. The 10-meter diameter target chamber on the right side of the illustration sets the scale for the facility

After exiting the main laser amplifier section, the beam passes through a spatial filter to remove any high-frequency noise that has accumulated on the wavefront of the pulse. The beam then enters the Final Optics Assembly (FOA) as it continues toward the target chamber. At this point along the propagation path, the wavelength of the pulse is $1.055 \mu \mathrm{m}$ (infrared) corresponding to the lasing transition wavelength in the Nd glass. It is in the FOA that the beam is frequency converted to $351-\mathrm{nm}(0.351 \mu \mathrm{m})$ by way of third-harmonic generator. The NIF frequency converter consists of a KDP plate and a deuterated KDP plate. The first crystal frequency doubles the $1.053 \mu \mathrm{m}$ light, while the second crystal combines first and second harmonic light to generate 3rd harmonic light at $0.351 \mu \mathrm{m}$. A final focus lens in the FOA focuses the beam from its nominal $35 \times 35 \mathrm{~cm}$ beam size, to a few hundred-micron diameter at the center of the target chamber approximately 8 meters away. The beams from all 192 beamlines must be carefully aligned and timed so that the energy hits a target at essentially the same time.

The commissioning of the first quad of NIF began late in 2002. The beamlines in the first quad were initially built out to contain 18 amplifier slabs, with an 11/7 configuration in the main and power amplifier sections, respectively. The initial lasers tests were designed to characterize the performance of the MOR and PAM, collectively called the Injection Laser System (ILS). Beginning in December 2002, the first low energy, unconverted laser pulses (with output energy of approximately 1-kJ per beamline of $1.05 \mu \mathrm{m}$ light) were conducted on NIF's four beamlines. During the first year of commissioning, the NIF operations team has conducted the first third harmonic $(3 \omega)$ shots, and operated at increasingly higher energies and power at $1 \omega, 2 \omega$ and $3 \omega$. In addition, NIF has already been used to conduct target experiment and target diagnostic commissioning activities. The LPOM was deployed in the NIF facility prior to the first shots in the commissioning series, and was used to set up the initial full system shot. Since then, LPOM has been used for every 
shot over the past year - approximately 200 full system shots with all four beamlines participating on most shots. The LPOM has proved to be an invaluable tool for the commissioning activities, providing accurate output power and energy settings over a wide range of operating conditions.

\section{DESCRIPTION OF THE LASER PERFORMANCE OPERATIONS MODEL}

The LPOM is an essential tool used by the NIF Shot Director to set up and assess the performance of each of NIF's laser beams. Its primary functions are to calculate settings for the lasers and diagnostics (shot setup), to assess operational and performance feasibility (equipment protection) and to analyze and archive laser performance (shot reporting). The LPOM has been implemented as one of the high-level systems of the Integrated Computer Control System (ICCS). For its first deployment, LPOM conducts its shot preparation activities outside of the shot life cycle. Interaction with the controls network is done manually through a database transfer of shot goals to and calculated shot setting from LPOM. Shot calculations are conducted many hours prior to the initiation of a shot; in the future, LPOM will be directly integrated into ICCS so that its calculational functions will be automated and controlled through a shot supervisor within the shot life cycle. These functions of the LPOM, as well as any computational activities needed to support commissioning and operations (such as assessment of off-normal operating conditions) are performed on an LPOM workstation within the NIF control room.

\subsection{Shot setup}

The primary role of the LPOM is to setup and optimize the performance of the NIF laser beams. LPOM starts the beam setup process based on requests of desired energies and the temporal shapes (power) of the beams at a specified location. During the Operations Phase, the specified location will normally be the Target Chamber Center. However, during Commissioning, experimenters need to specify energies at other locations along the NIF beamline activities to assist in characterizing system performance. In addition, the ability to specify output pulses at $1 \omega(1.053-\mu \mathrm{m}), 2 \omega(526.5-\mathrm{nm})$ or $3 \omega(351.5-\mathrm{nm})$ will be supported. To perform its predictive function, the LPOM incorporates a model of the laser system having enough fidelity to produce realistic and accurate performance calculations. The model includes the optical paths and configurations for each beam, spatial pulse shaping effects, energy control, amplifier gains, transmission losses, and frequency conversion. For useful and accurate predictions, the laser model must be kept current with the "as built" state of the laser system. The LPOM therefore continually acquires data from past shots in order to update its energetics models (gains and losses). Eventually, the LPOM will be connected to the metrology and maintenance databases so that information on component changeouts, as well as measured metrology data for installed optical elements can be automatically incorporated into the modeling.

Figure 2 illustrates the LPOM shot setup process. LPOM reads the goals of an experiment or NIF shot from the ICCS Setup Database. LPOM takes the goals of the experiment, including information about the proposed laser configuration (e.g., the number of amplifier slabs to be used, the level of flashlamp pumping of the slabs), and generates beamline specific input files for PROP. LPOM conducts a series of PROP calculations in order to self-consistently determine the settings for the ILS system that will produce the required output energetics. The energetics calculations for each beamline in a quad can be run independently on separate CPU's (the current system consists of $22.8-\mathrm{GHz}$, dual processor boxes); however, the calculations for the components of the single PAM feeding the quad can only be run in series. Currently, a complete calculation of setup of the entire quad takes approximately 3 minutes, using a spatial grid of 128x128 (1-mm resolution in the main laser) and 10 temporal slices (approximately 0.5-ns resolution). Upon completion of its setup calculation, the LPOM has determined: 1) the waveplate settings required by the PABTS splitting waveplates; 2) the attenuations for the input and output waveplates on the PAM four-pass amplifier; and 3) the MOR temporal pulse shape. In addition, LPOM predicts the energy and power expected at each diagnostic location for the quad. These energies are used to determine the attenuation settings necessary for each diagnostic device so that the shot is accurately measured. These settings are uploaded to the ICCS Shot database, where they can be accessed by all NIF subsystems (ILS, and Laser Diagnostics) that require this data in order to set up for a shot. At this point, all systems are ready to implement these settings, in preparation for a shot.

In order to maintain an accurate model of each beamline, LPOM requires feedback at the conclusion of each shot from each diagnostic. This feedback loop is shown in Figure 3. When the predictions of the model begin to deviate from measured data, LPOM uses a set of measured data to modify or optimize its model of the laser. Currently, this updating 
or optimization is done manually; a non-linear optimization package has been designed for future use when more beamlines will be employed. Commissioning experience has shown that convergence of the LPOM main laser model can be achieved with the modification of only two parameters (a multiplier on the average small signal gain, and a distributed transmission loss).

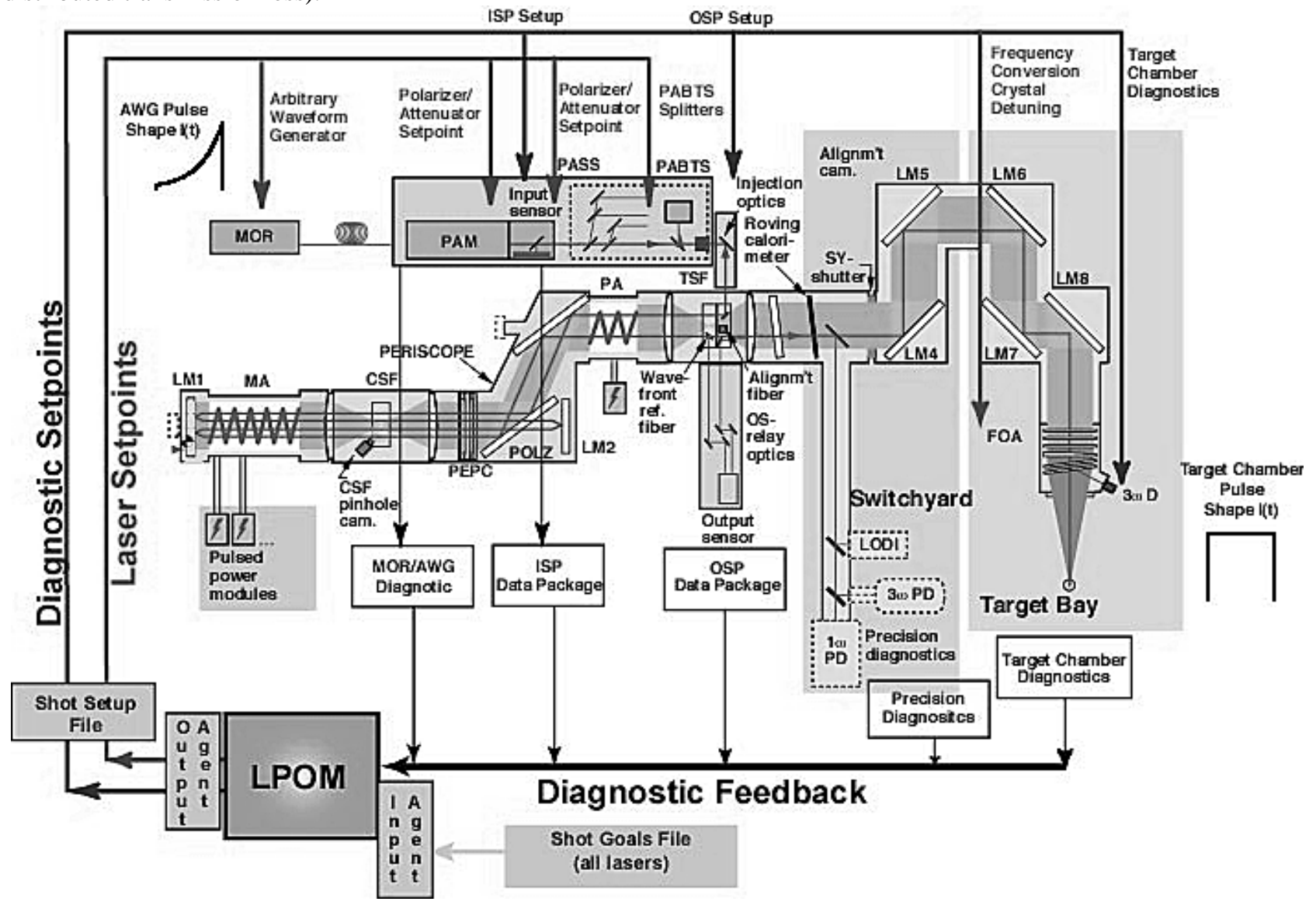

Figure 2- The LPOM Feedback loop. The illustration shows the process of predicting the laser equipment and laser diagnostics setpoints, and receiving the measured diagnostic data. In an iterative mode of operation, the measured data would be used to adjust the model, leading to different setpoints for subsequent shots

\subsection{Equipment Protection}

The Equipment Protection Module of the LPOM is designed to help minimize the risk of optical damage to the system. This module will protect the ILS's 4-pass amplifier, the PABTS optics, the main amplifier and the Final Optics Assembly (FOA). The Equipment Protection Module has two components that play roles at different phases in the Shot Cycle. The first module, called the Setup Assessment Code, checks the calculated system setup before the ICCS Laser Supervisory System implements it. The second module, called the Setup Verification Module, evaluates the actual pulses generated during a series of low-energy shots prior to the initiation of a full-system shot. Its role is to verify that the ILS pulses match those predicted and that these pulses will not have a significant negative impact on equipment lifetime. Both modules serve as administrative controls for equipment protection, with the Assessment Module serving as a first line of defense and the Verification Module serving as a next line of defense.

The Setup Assessment module evaluates the feasibility of the proposed shot setup calculated by the Shot Setup Module. In this context, "feasibility" has two components. First, the impact of the shot on equipment protection or equipment lifetime must be assessed. Second, the ability of the NIF Laser system to meet the goals or needs (e.g., total energy delivered to the chamber, power imbalance) of a proposed experiment must be assessed. The Shot Setup module uses the energetics calculations performed in Shot Setup to evaluate the probability of damage to key optical elements in the 
NIF beamline. These tests include the probability of optical damage initiation, probability of beam filamentation, and the probability of excessive beam spatial contrast. If the proposed system setup does not exceed any of these criteria, the LPOM's recommendations of laser system and laser diagnostic system settings are transmitted to the ICCS Laser Supervisory System and the Shot Director is notified that the system can support the proposed shot. If either criteria (equipment protection, or inability to meet the goals) is not satisfied, alerts are sent to the ICCS Laser Supervisory System and Shot Director.

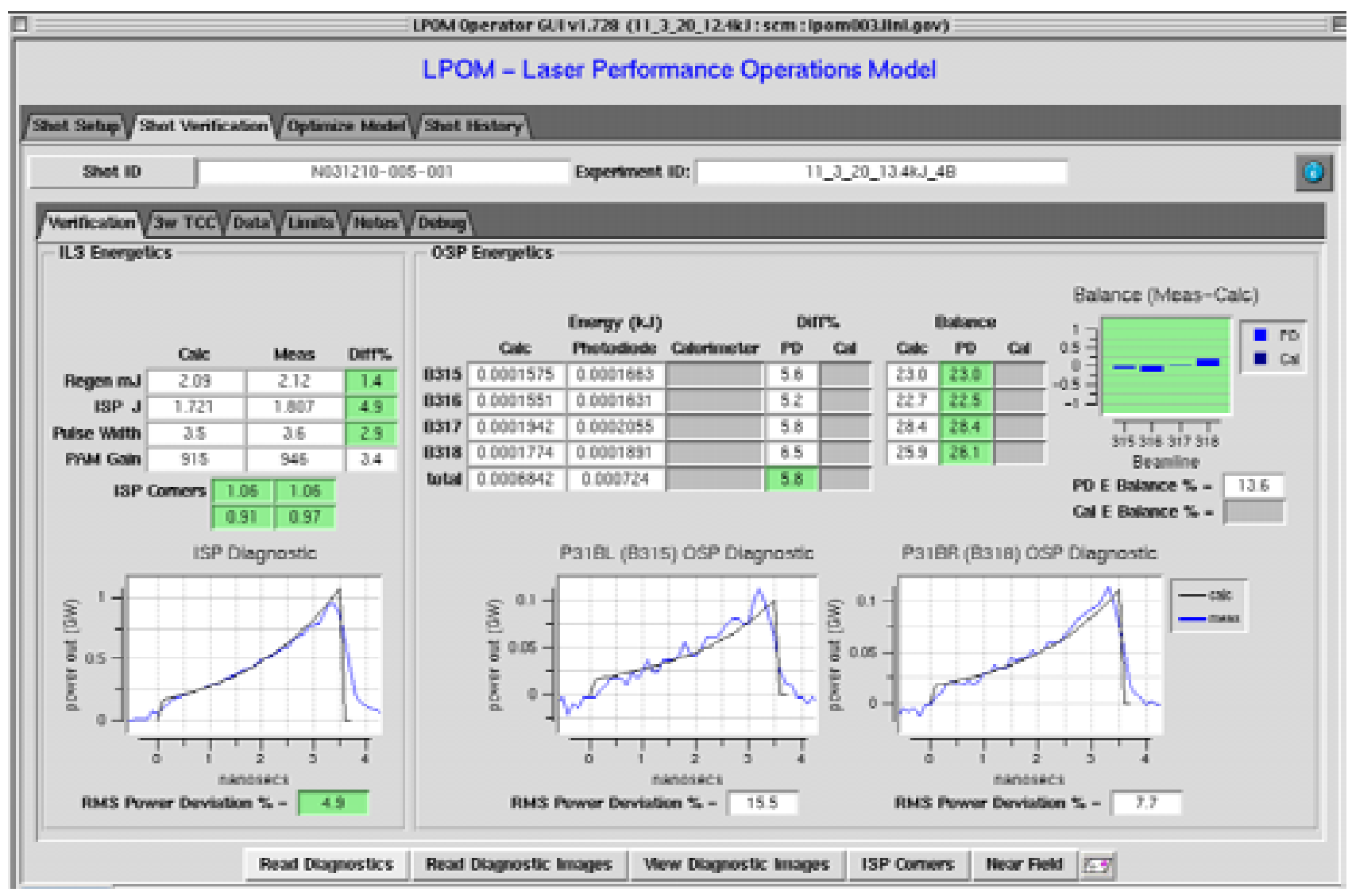

Figure 3 - Graphical capture of the LPOM Shot Verification Screen on the LPOM GUI. This screen shows the comparison of various predicted and measured quantities after a rod shot. The system is declared to be ready for a full system shot if all of the performance metrics are within prescribed limits.

The Setup Verification Code is tasked with verifying that the actual laser pulse and energy output from the ILS will not damage the main laser or final optics. To achieve this, the Setup Verification Code will accumulate measurements of the pulse energy, power and spatial shape taken during a series of low-energy "rod" shots prior to the full system shot. The module compares the measured ILS power to the predicted power, and evaluates the statistical variations in both pulse energy and temporal shape. In addition, LPOM compares the measured near field spatial profile to that predicted, and verifies that the energy splits among the beamlines of a quad agrees with the requested setpoint. Using rules or guidelines provided by the NIF Program, the Setup Verification Code (through the Shot Director) can stop the Shot Cycle if there is a high probability of significant equipment damage or if there is significant deviation from predicted results. If the measured rod shot energies are within the allowable operating range, an alert, along with a graphical summary, is sent to the Shot Director that the system is prepared for a primary shot. Figure 3 shows a screen capture of 'Shot Verification Screen' on the LPOM GUI at the end of a rod shot. Metrics that fall inside or outside of prescribed limits are highlighted with green and red, respectively. 


\subsection{Data Analysis and Reporting}

The third function of the LPOM is to provide post-shot data analysis and reporting. The LPOM is directly linked to the ICCS shot database. After a shot is conducted, the LPOM operator can request access to the data (energetics, near-field and far-field images). The LPOM manipulates the measured data, using routines written in IDL, to present comparisons of predicted and measured results. In particular, LPOM can quickly (within minutes) provide the NIF Shot Director and user community with a report that summarizes the measured energetics of a shot, and gives metrics relating the ability to match the requested goals of the shot. In addition, the LPOM data reporting system also can access and display nearfield and far-field images taken on each of the laser diagnostic locations, and provide comparisons with predicted images. The results of the post-shot analysis are displayed on the LPOM GUI, while a subset of the analysis is presented to the Shot Director through a Shot Supervisor. The 'Data Analysis Screen' on the LPOM GUI is an extension of that shown in Figure 3, allowing for information and access to data that is related to only system shots. Future work is planned on presenting this type of data at a much higher level to accommodate post shot reporting for hundreds of beamlines (compared to the current four beamlines).

\section{RESULTS}

The initial main laser shot campaign during the NIF commissioning activities consisted of $61 \omega$ shots starting at low energy (1-kJ per beamline) and concluding at an energy that was approximately half of the NIF operating point (10-kJ per beamline). LPOM was used to determine the settings of the initial 1-kJ shot, making its predictions based on expected optical transmissions and calculated gain profiles. For the initial shot, the LPOM's predicted PABTS waveplates were ignored, choosing rather to set the waveplates to inject equal energy down each beamline. The total output energy $(3.12 \mathrm{~kJ})$ for the first shot was low by approximately $20 \%$, and the balance among the 4 beams was $18 \%$ (i.e., the energy for one of the 4 beamlines was $18 \%$ lower than the mean of the quad). After a $2^{\text {nd }} 1-\mathrm{kJ}$ shot, the LPOM beamline model was adjusted to match the measured net gain, while keeping fixed its assumed chain transmission. For the next four shots in the campaign $(3,5,7$ and $11 \mathrm{~kJ}$ per beamline), the measured total output energy ranged from 2-6\% of requested, and the energy balance among beamlines was approximately $1 \%$. This means that the energy of any single beamline was within $1 \%$ of the energy of all of the beamlines in a quad.

Over the past six months, we have taken a large number of higher energy shots with output pulses at $1 \omega, 2 \omega$ and $3 \omega$. Figure 4 shows a comparison of the measured mean laser beamline $1 \omega$ energies to those predicted by LPOM. For these shots, the beamlines (numbered 315-318) were operated in an 11/7-amplifier slab configuration, with an explosion fraction of $18 \%$. Pulse durations ranged from 5 to $24.5 \mathrm{~ns}$, with requested output shapes that were either flat in time, or highly shaped ignition pulses. This comparison shows that the LPOM is extremely accurate over a large operating range, covering almost the entire saturation curve. In addition, one can see that the measured output energies for the four beamlines are approximately equal for all shots taken. Since the net gains for the beamlines differ by almost $50 \%$ at low energy (no saturation), this high level of energy balance among the 4 beamlines is impressive. This illustrates LPOM's ability to model the beamlines independently, and to accurately determine the required PABTS waveplate settings to achieve the required injection energy balance.

By relying on detailed beamline models, the LPOM has been capable to accurately model the energetics for many of the different operating conditions tested during commissioning. We have recently concluded a series of shots with the laser configured in an 11/3 geometry, and the slabs pumped at a $20 \%$ explosion fraction. Using relationships derived from detailed offline gain modeling, we scaled the slab gains from the $18 \%$ value to a $20 \%$ value. Also, the increased chain transmission arising from the removal of 4 slabs in the PA (8 total slab passes) was automatically accounted for by LPOM in the construction of the PROP input decks. After the first shot in the series (a low energy, 1-kJ, calibration 


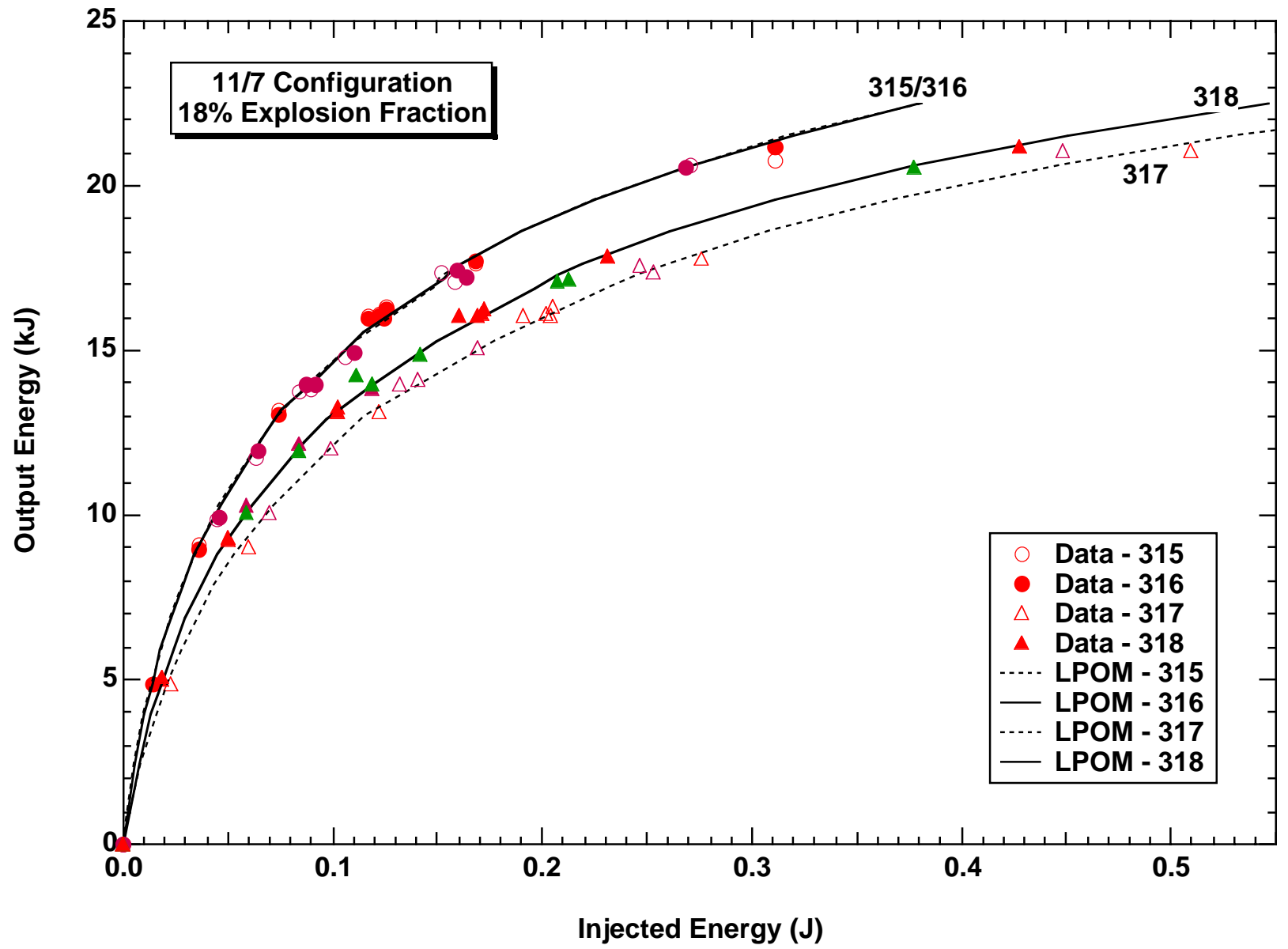

Figure 4 - Comparison of predicted output $1 \omega$ energy (lines) vs. measured for the four NIF beamlines. For this series of shots, the laser was operated at an explosion fraction of $18 \%$, with an 18 laser slabs (11 in the MA, and 7 in the PA).

shot), the gain multiplier was adjusted slightly for beamline 315, to account for $4 \%$ discrepancy in predicted performance. Figure 5 shows the comparison of measured energy to that predicted with the recalibrated LPOM model for the remaining shots in the series. The agreement for all four beamlines over a significant energy range is extremely good. This not only shows the robustness of the LPOM predictive capabilities over significant operating ranges and configurations, but also shows the near automation of LPOM to model these different conditions. The logic of LPOM is such that it modifies its models automatically based on the setup file read from the ICCS database. For almost all shots taken during commissioning, no operator intervention has been required to setup different laser configurations.

An accurate prediction of output energy is only one measure of the LPOM's modeling capabilities. The other important measure is the temporal shape, or power, of the output pulse. Since the pulse undergoes twelve orders of magnitude amplification from the MOR to laser output, the initial temporal shape must be extremely accurate in order to match the requested shape. Figure 6 shows the comparison of the output power (right) for a high-energy $(20-\mathrm{kJ})$, 1 $1 \omega \mathrm{pulse}$. The graph on the left shows the requested injection pulse shape, illustrating the large amount of temporal shaping required to 


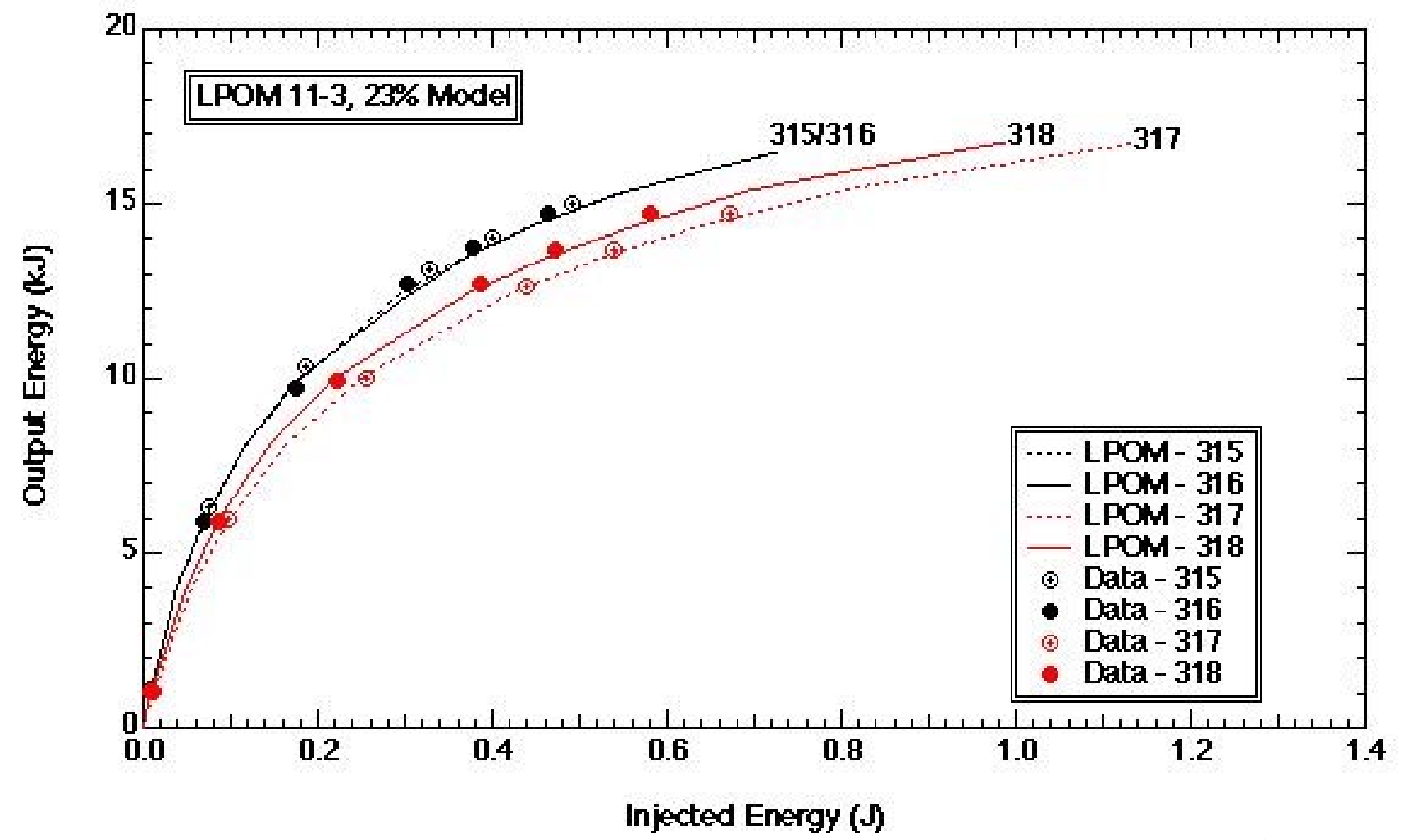

Figure 5 - Comparison of predicted output $1 \omega$ energy (lines) vs. measured for the four NIF beamlines. For this series of shots, the laser was operated at an explosion fraction of $20 \%$, with a 14 laser slabs (11 in the MA, and 3 in the PA).

compensate for gain saturation. The agreement is very good, further illustrating the accuracy of the LPOM energetics model. Figure 7 shows the comparison of a measured high energy, $2 \omega$ ignition pulse with the requested shape. The
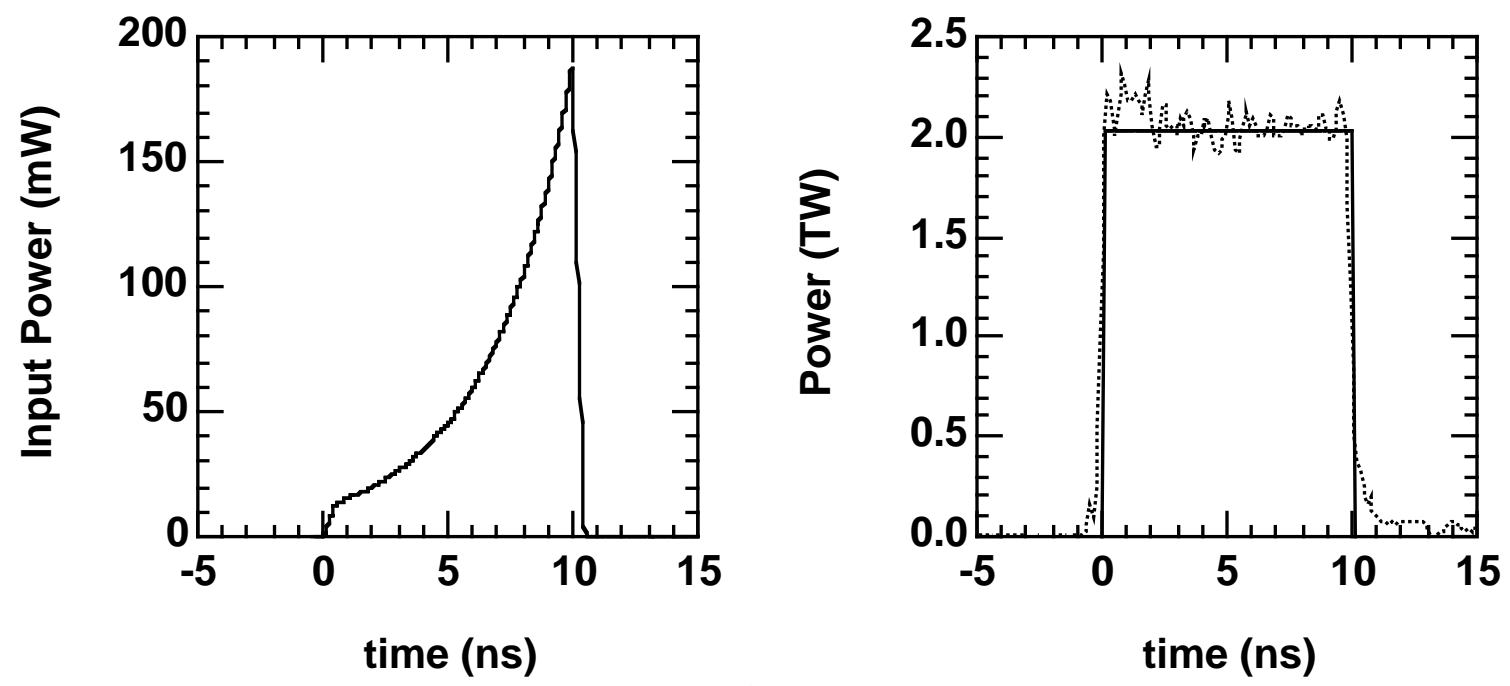

Figure 6 - The graph on the right shows a comparison of measured (dashed line) to requested output power (solid) for a 21-kJ per beamline NIF shot. The figure on the left is the MOR input pulse shape required to produce the flat-in-time output pulse.

initial MOR pulse shape in on the left side of the figure. The ability to produce a frequency-converted pulse shape with this high level of shaping without any adjustments to the model or hardware is a testament to both the LPOM's modeling capabilities and to the flexibility of the ILS pulse generation system. This level of accuracy in predicting both energy and 
power can only be achieved if the models properly account for the contribution of gain and optical losses to the net energy gain of a beamline.
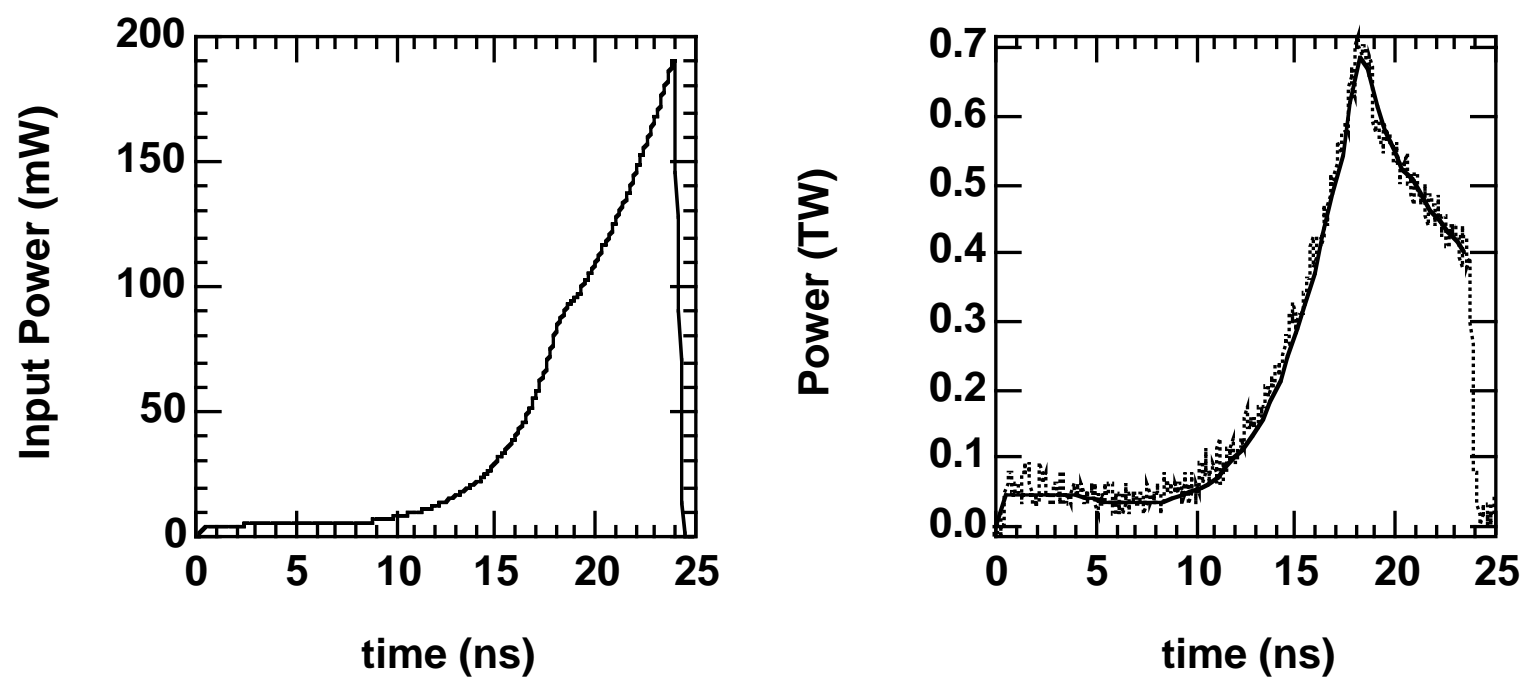

Figure 7 - The graph on the right shows a comparison of measured (dashed line) to requested output power (solid) for a high energy $(5.1-\mathrm{kJ}), 2 \omega$ ignition pulse test on NIF. The figure on the left is the MOR input pulse shape required to produce the highly-shaped output pulse.

The NIF frequency conversion system has been operated up to energies of 10-kJ per beamline, making it the highest energy blue laser system in the world ${ }^{7}$. Figure 8 shows a comparison of the measured and predicted $3 \omega$ conversion efficiency vs. $1 \omega$ energy. Again, the agreement over such a large range of operating conditions is very impressive. All of the data presented here is for 3.5-ns, flat in time output pulses. The robustness of the LPOM $1 \omega$ and $3 \omega$ models gives us great confidence that we will be able to accurately produce highly-shaped $3 \omega$ temporal pulses required for ignition experiments, while meeting the extreme energy and power balance required placed upon NIF.

\section{SUMMARY}

We have described a computational system, LPOM, which is used to automate the laser setup and diagnosis of the National Ignition Facility. The LPOM uses an accurate, detailed physics models of beam propagation and energy extraction, coupled with descriptions of the individual optical configuration of each beamline to produce the settings required on the injection laser system that will produce the requested output energetics. The LPOM was deployed prior to the NIF commissioning activities, and interacts with the NIF controls system by interaction with the shot database. The LPOM has been used to set up every shot conducted on NIF (over 200 at this point), and has been able to produce output pulses that match those requested within a few percent. The ability to meet requested energy and power, and to achieve beam-to-beam energy balance with great accurate is unparalleled for any large, multibeam laser fusion system. In addition, the LPOM provides an online equipment protection function that minimizes the risk that a proposed shot can cause significant optical damage to the system. This equipment protection feature is evoked both prior to the initiation of a shot (accessing the safety of the requested shot), and during the actual system shot cycle (verifying that the injection laser system is correctly setup to produce the requested energies and powers). Finally, the LPOM provides shot reporting and data analysis that gives a detailed verification of a shot within minutes of its completion. The ability of this system to automate the setup, analysis and reports of shots in the first NIF quad well is one of the first critical steps in making the complete NIF facility an essential tool for stockpile stewardship. 


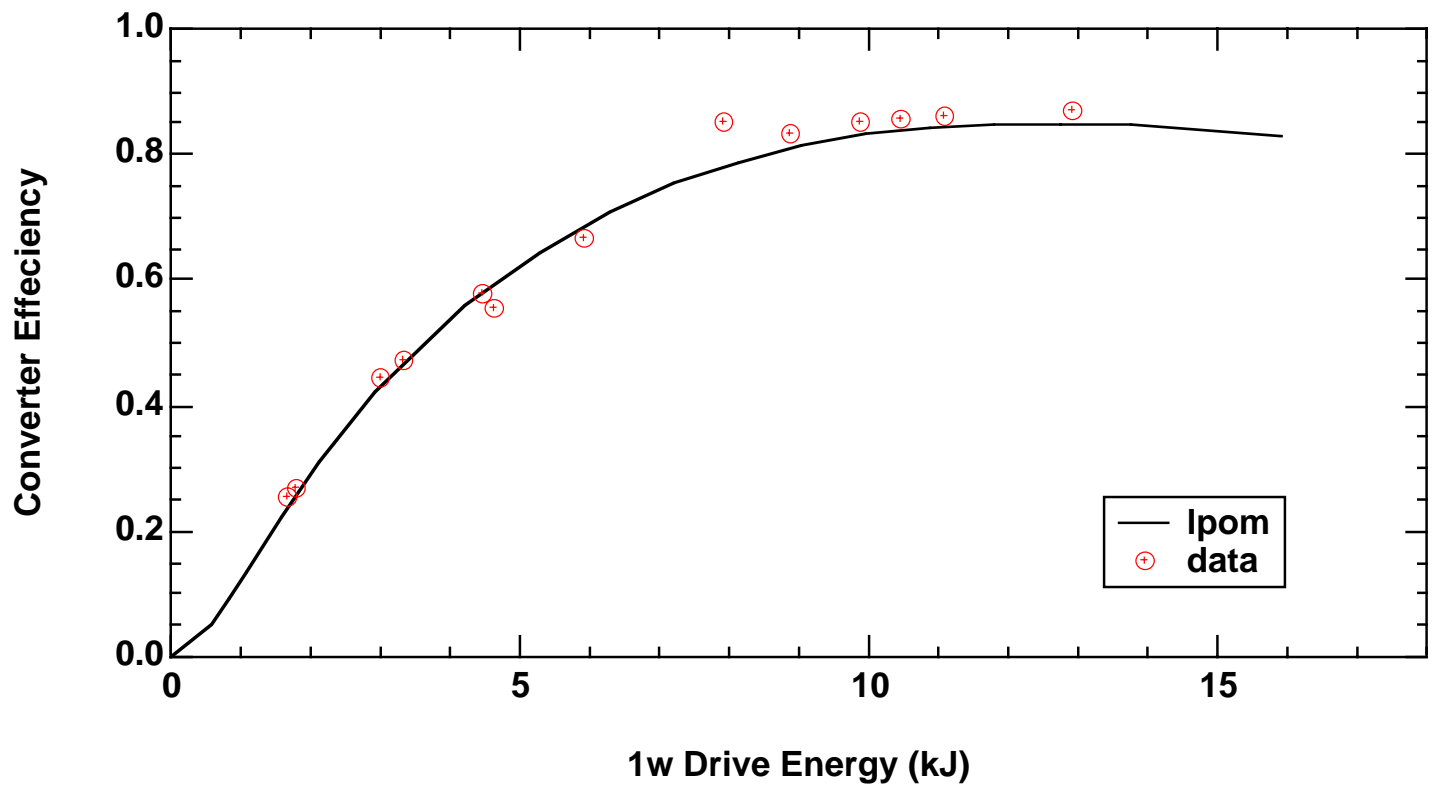

Figure 8 - Comparison of predicted and measured $3 \omega$ conversion efficiency for the first series of full system shots on NIF. The pulses for these shots were all 3.5-ns flat-in-time at output.

\section{REFERENCES}

1. W.J. Hogan, E. I. Moses, B. E. Warner, et. al., “The National Ignition Facility”, Nucl. Fusion, 41(5), pp 567-573 (2001)

2. C. A. Haynam, R. A. Sacks, and M. J. Shaw, "Computational modeling in support of the National Ignition Facility",presented at International Conference on Accelerator and Large Experimental Physics and Controls Systems (ICALEPS) (2001)

3. M. Shaw, W. Williams, K. Jancaitis, C. Widmayer, and R. House, "Performance and Operational Modling of the National Ignition Facility', at International Symposium on Optical Science and Technology (2003)

4. J. T. Hunt, K. R. Manes, J. R. Murray, et. al., "Laser design basis for the National Ignition Facility", Fusion Technol, 26, pp 767-771 (1994)

5. L. M. Frantz and J. S. Nodvik, "Theory of pulse propagation in a laser amplifier", J. Appl. Phys., 34, 2346-2349 (1963)

6. M. A. Rhodes, B. Woods, J. J. Deyoreo, D. Roberts, L. J. Atherton, "Performance of large-aperture optical switches for high-energy inertial-confinement fusion lasers", Applied Optics, 34, pp 5312-5325 (1995)

7. P. J. Wegner, "Frequency Conversion Performance of the NIF laser system", presented at the $23^{\text {rd }}$ Annual Conference on Lasers and Electooptics (2003).

This work was performed under the auspices of the U.S. Department of Energy by University of California, Lawrence Livermore National Laboratory under contract No. W-7405-Eng-48. 